\title{
UTILIZAÇÃO DE SIMULAÇÃO COMPUTACIONAL PARA O ESTUDO DAS PROPRIEDADES ELETROMAGNÉTICAS DE UM COMPÓSITO MAGNÉTICO MACIO (SMC) PARA FUTURAS APLICAÇÕES EM MOTORES DE INDUÇÃO
}

\author{
Jaime Back ${ }^{1-2}$, Juliano Schirmbeck ${ }^{1}$, Jeferson Gaio ${ }^{2}$, Lírio Schaeffer ${ }^{2}$ \\ ${ }^{1}$ Curso de Engenharia Elétrica, Universidade do Vale do Taquari, CEP 95914-014, Lajeado, Rio Grande do Sul, Brasil. \\ 2 Programa de Pós-Graduação em Engenharia de Minas, Metalúrgica e de Materiais - PPGE3M, Universidade Federal do Rio \\ Grande do Sul, CEP 91501-970, Porto Alegre, Rio Grande do Sul, Brasil.
}

*E-mail: jaime.back@gmail.com

\section{RESUMO}

Este trabalho apresenta o desenvolvimento de um método de simulação computacional para o estudo das propriedades eletromagnéticas de um material compósito para aplicação em motores de indução (rotor e estator), motivado pela possibilidade de aplicação dos materiais SMCs (Soft Magnetic Composites) como alternativa aos aços elétricos tradicionais (laminado de grão não orientado). Desta forma, objetivou-se o desenvolvimento de um ambiente de simulação computacional afim de facilitar o levantamento dos parâmetros elétricos e magnéticos de um motor de indução, sem a necessidade da construção de um modelo físico. Para esta tarefa, utilizou-se o software FEMM 4.2 para as simulações do modelo em 2D, bem como para validar os parâmetros coletados em comparação aos materiais comerciais de mesma composição. Uma relação entre a circulação de fluxo magnético nos materiais SMCs e nas chapas laminadas foi feita, na qual observou-se que a amostra de material compósito possuía propriedades compatíveis aos aços laminados, porém uma resistividade elétrica maior devido a camada isolante das partículas ser mais espessa. Como resultado final, pode-se levantar um método de simulação de forma a facilitar a pesquisa e desenvolvimento de diferentes ligas de pó de Fe, que poderão utilizadas em novas topologias de motores elétricos de indução, para as mais diferentes aplicações industriais.

Palavras-chave: Materiais Magnéticos Macios. Motores de indução. Fluxo magnético.

\section{Introdução}

Nos últimos anos os compósitos ferromagnéticos moles, também conhecidos como Soft Magnetic Composites (SMC) estão sendo amplamente estudados devido às muitas vantagens que oferecem sobre as chapas de aço eletromagnéticas em relação às suas propriedades magnéticas isotrópicas, alta resistividade elétrica, design flexível, potencial para redução de tamanho e alta flexibilidade de projeto [1]. Para usar núcleos magnéticos em pó nas aplicações de campo magnético CA (Corrente Alternada), no entanto, é importante reduzir a perda de ferro, isto é, a soma da perda de corrente de Foucault e perda de histerese.

Os núcleos magnéticos em pó são, portanto, fabricados pela compactação de partículas magnéticas em pó e revestimento com camadas isolantes para evitar a formação de correntes parasitas. Esta técnica de revestimento com uma estrutura de microencapsulamento como isolamento é crucial para melhorar as propriedades magnéticas de um núcleo para motores $\mathrm{CA}$. Tecnologias e processos mais modernos permitem que as propriedades desses materiais sejam melhoradas para prover propriedades magnéticas competitivas (alta permeabilidade e magnetização de saturação), mas com alta resistividade elétrica visando minimizar as perdas clássicas por correntes parasitas [2].

Ainda segundo Shokrollahi e Janghorban [2] natureza isotrópica do SMC combinada às possibilidades de conformação permite o planejamento de peças com geometrias não usuais e tridimensionais, oferecendo vantagens em relação aos aços elétricos laminados tradicionais. Assim, ligas de materiais magnéticos macios, compostos de pós de ferro, em conjunto a outros elementos, tais como fósforo, silício ou níquel, poderão ser utilizadas em motores de núcleos maciços, construídos a partir de chapas de aço laminadas [3-4].

Neste contexto, partículas magnéticas, tais como cobalto, ferro, ferritas como $\mathrm{Fe} 3 \mathrm{O} 4$ e $\gamma$-Fe2O3, entre outros óxidos têm mostrado potenciais aplicações em diversos campos, incluindo ferrofluidos, aplicações industriais, robótica, remediação ambiental, áreas biomédicas no diagnóstico e terapia, como ressonância magnética nuclear, entre outas aplicações [5-6]. 
Os parâmetros magnéticos comumente medidos são obtidos a partir de uma curva de magnetização em função do campo aplicado. A aplicação de um campo magnético suficientemente grande causa o alinhamento dos spins do material com o campo. Com a diminuição do campo, os spins cessam seu alinhamento e a magnetização decresce [6].

A Figura 1 mostra as regiões de aplicação para os materiais utilizados em campo magnético AC. Aços-silício laminados tem uma alta permeabilidade, porém suas perdas são aumentadas em função do aumento da frequência. A Ferrite mole tem uma baixa perda magnética para altas frequências, porém sua baixa permeabilidade exige a utilização de muito material. Já os SMCs cobrem uma região intermediária de aplicação.

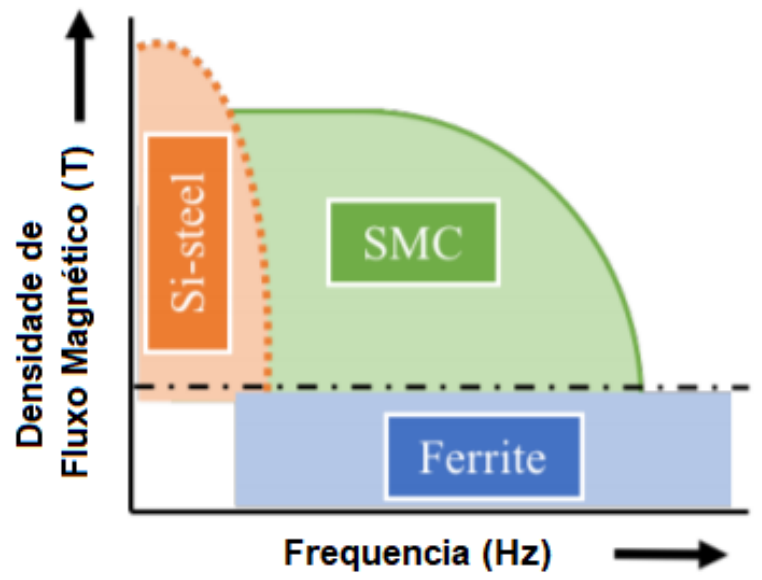

Figura 1 - Regiões de aplicação dos materiais em CA [1].

A motivação para pesquisa ocorre visto que atualmente as máquinas elétricas são construídas a partir de núcleos ou pacotes compostos de chapas de aço magnético, laminadas e justapostas, circundados por um enrolamento de fio de cobre esmaltado. O processo de fabricação destas máquinas elétricas é, desta forma, mais caro se comparados com o processo de fundição, por exemplo, processo que resultaria em núcleos maciços [7], já que novas perspectivas indicam que ligas de materiais magnéticos macios, obtidos a partir de pó de ferro base, misturados com outros elementos, poderão apresentar propriedades superiores aos obtido a partir de chapas de aço laminadas.

Os materiais macios sinterizados, produzidos por $\mathrm{M} / \mathrm{P}$, são alvo de diferentes estudos para suas aplicações, na eletrônica e principalmente em motores elétricos, devido à grande parcela de energia elétrica que eles consomem [3,7-11]. Uma diminuição nas perdas, mesmo que pequena, resultaria em uma economia substancial considerando um consumo total a médio e longo prazo.

As maiores vantagens de se utilizar materiais sinterizados são o número reduzido de etapas no processo de fabricação dos núcleos, resultando em menor gasto de energia no processo de fabricação, além da matéria prima ser de menor custo comparada a chapas laminadas.

\section{Referencial Teórico}

Esta seção apresenta uma breve revisão sobre os fundamentos do magnetismo, a classificação de materiais magnéticos com ênfase em materiais ferromagnéticos. São também discutidas as perdas em aplicações eletromagnéticas. Uma introdução ao processo de metalurgia do pó é feita, assim como os SMCs, destacando suas propriedades, vantagens e desvantagens em relação a outros materiais magnéticos moles e suas aplicações.

\subsection{Magnetismo}

Todos os fenômenos magnéticos são devidos às cargas elétricas que estão em movimento. Analisando uma porção microscópica de material magnético se encontraria elétrons orbitando em volta dos núcleos e elétrons girando pelo seu próprio eixo. Em virtude do pequeno tamanho dessas espiras de corrente elétrica trata-se elas como dipolos magnéticos.

Quando um campo magnético é aplicado nestas espiras, ocorre o alinhamento líquido dos dipolos magnéticos e o meio se torna magnetizado. A polarização elétrica se dá na direção do campo elétrico $E$. Materiais que possuem magnetização paralela ao campo magnético $B$ são chamados de paramagnéticos. Já materiais que possuem magnetização contrária ao campo magnético $B$ são chamados de ferromagnéticos [12]. Assim, de acordo com a lei de Ampére, a corrente elétrica $I$ produz, além deste campo elétrico, um campo magnético $H$.

\subsection{Materiais Ferromagnéticos}

Os materiais ferromagnéticos são aqueles que, ao serem submetidos a um campo magnético externo, apresentam uma magnetização resultante muito mais intensa que o campo aplicado e ainda mantém alguma magnetização remanente mesmo após o campo externo ser removido [13]. Este comportamento pode ser observado na forma de um laço de histerese na curva de magnetização B.H como mostrada na Figura 2. 


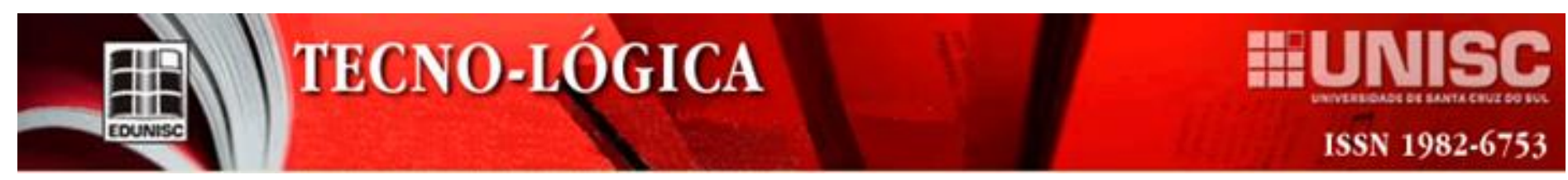

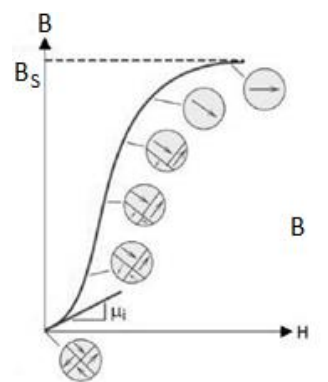

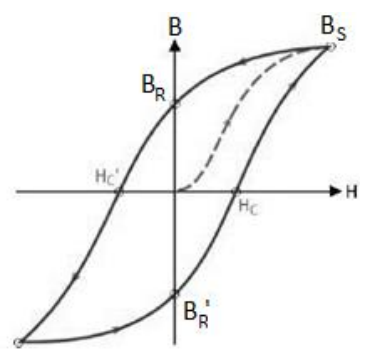

Figura 2 - (a) Curva inicial de magnetização em um grão de material ferromagnético. (b) Ciclo de histerese para um material ferromagnético inicialmente desmagnetizado. Adaptado de [13].

\subsection{Perdas Magnéticas}

Os materiais ferromagnéticos são aqueles que, ao serem submetidos a um campo magnético externo, apresentam uma magnetização resultante muito mais intensa que o campo aplicado e ainda mantém alguma magnetização remanente mesmo após o campo externo ser removido [13-14]. Este comportamento pode ser observado na forma de um laço de histerese na curva de magnetização B.H como mostrada na Figura 2.

Correntes parasitas (eddy currents), também conhecidas como correntes de Foucault, são induzidas em qualquer material condutor por um fluxo magnético alternado [14]. Considerando um campo alternado e um material uniforme (desconsiderando efeitos de desmagnetização, efeito pelicular, entre outros efeitos estruturais) as correntes ocorrerão como mostrado na Figura 3, perpendicularmente a direção do campo magnético variante.

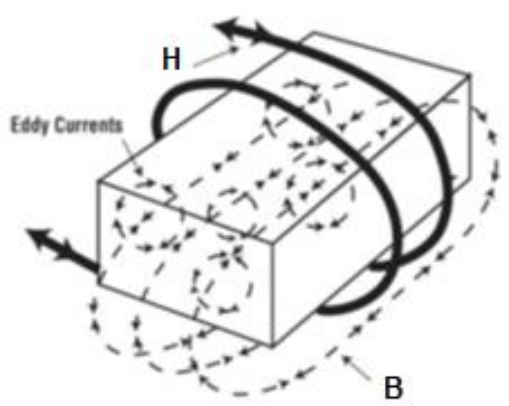

Figura 3 - Ilustração de como as correntes parasitas são geradas em um material condutor. Adaptado de [14].

Já a Figura 4 apresenta o efeito das correntes parasitas em dois materiais de espessuras diferentes. Em materiais maciços haverá uma maior propagação destas correntes, como mostrado na figura a esquerda, o que causa perdas consideráveis. Em materiais laminados (figura a direita), haverá pouco espaço para as correntes parasitas se propagarem, devido à limitação estrutural do material e também do isolamento entre cada uma das chapas.

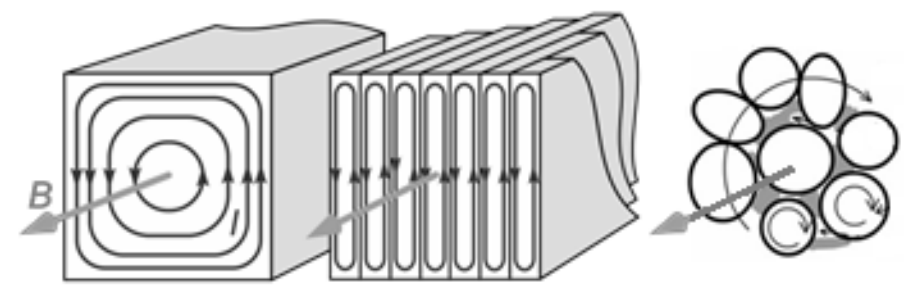

Figura 4 - Correntes parasitas em um material condutor maciço, em chapas isoladas e em um compósito magnético particulado. Adaptado de [14].

\subsection{Compósitos Magnéticos Macios}

Fabricados via metalurgia do pó, os materiais compósitos magnéticos macios são materiais com propriedades eletromagnéticas e que possuem dois ou mais elementos em sua composição. Estes materiais são gerados de minúsculas partículas de pó de ferro cobertas por um material dielétrico, que produz isolação elétrica aos grãos Figura 5.a. O pó de ferro é misturado com material dielétrico e lubrificante, como fósforo por exemplo.

Esta mistura é colocada em uma matriz e então compactada para gerar peças de material ferromagnético, como mostra a sequência da Figura 5-c. O método utilizado para compactação é a prensagem axial numa matriz de aço. Após este processo, o material é levado a um forno para fazer com que os grãos de ferro se misturem ao material dielétrico. Recomenda-se a utilização destes materiais para construção de núcleos de equipamentos eletromagnéticos sujeitos a campos magnéticos variáveis, por estes materiais possuírem isotropia magnética e alta resistividade elétrica $[2,11,15]$.

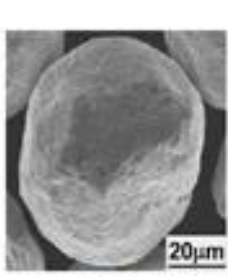

(a)

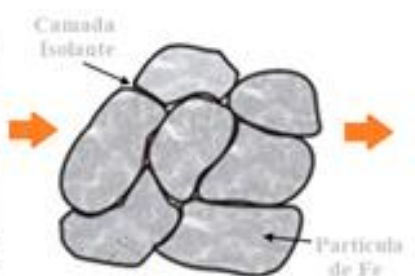

(b)

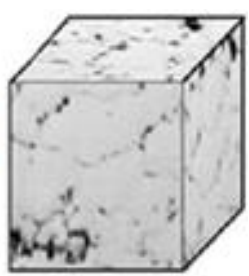

(c)
Figura 5 - Ilustração do processo de criação de um SMC.

$\mathrm{O}$ revestimento isolante que separa individualmente as partículas de pó de ferro em um produto SMC é a característica fundamental desta tecnologia. Sua espessura, cobertura e resistência sob as diferentes operações de processamento são aspectos fundamentais para as propriedades de uma peça magnética. $\mathrm{O}$ revestimento isolante tem como principal finalidade aumentar a resistividade e/ou atuação elétrica entre as partículas do material ferromagnético usado no SMC [2]. Na maioria dos casos, como material ferromagnético (núcleo da partícula de SMC) utiliza-se o ferro puro, ou pó de ferro baixa liga, com 


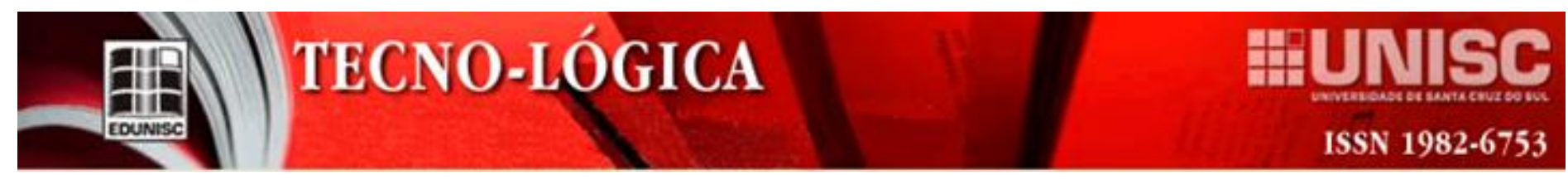

distribuição de tamanho de partículas variados, produzidos por meio de técnicas tradicionais de atomização em água.

A ilustração da Figura 6 apresenta uma partícula de ferro sendo recoberta por particulados de fosfato de estrôncio e óxido bórico. Após o processo térmico, é possível observar o surgimento de uma fina camada de dióxido de silício recobrindo a partícula de ferro [1-2].

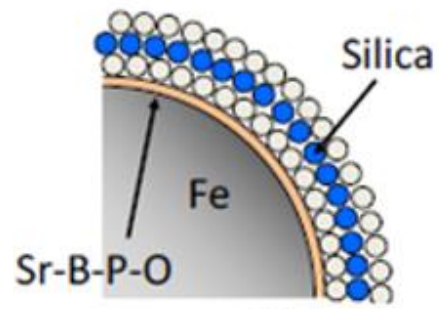

(a)

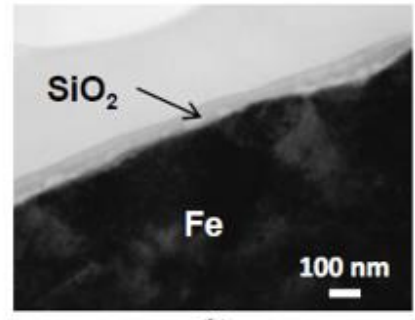

(b)
Figura 6 - (a) Ilustração da partícula de ferro sendo recoberta. (b) Fina camada de dióxido de silício recobrindo a partícula após tratamento térmico. Adaptado de [1].

\section{Parte Experimental e Metodológica}

\subsection{Caracterização eletromagnética do SMC}

A caracterização desses materiais pode ser feita por meio de corpos de prova, que proporcionaram as análises das propriedades físicas e magnéticas de cada material comparando estas com resultados existentes em literatura [3, 7-10].

Para o ensaio de histerese faz-se necessário a preparação da amostra, como apresentado na Figura 7, e depois na bobinagem (enrolamento de fios de cobre) de espiras primárias e secundárias no anel, conhecidas como Anel de Roland, de acordo com a norma ASTM A773/A 773M-01 [16].

O procedimento consiste em isolar o anel com filme plástico para evitar danos ao fio esmaltado. Em seguida, enrolar as espiras da bobina do secundário com fio AWG 26, contando o número de voltas. Para a bobina primária, deve-se efetuar um novo isolamento, então enrolar as espiras primárias com fio AWG 23, também contando o total de voltas dadas para esta bobina.

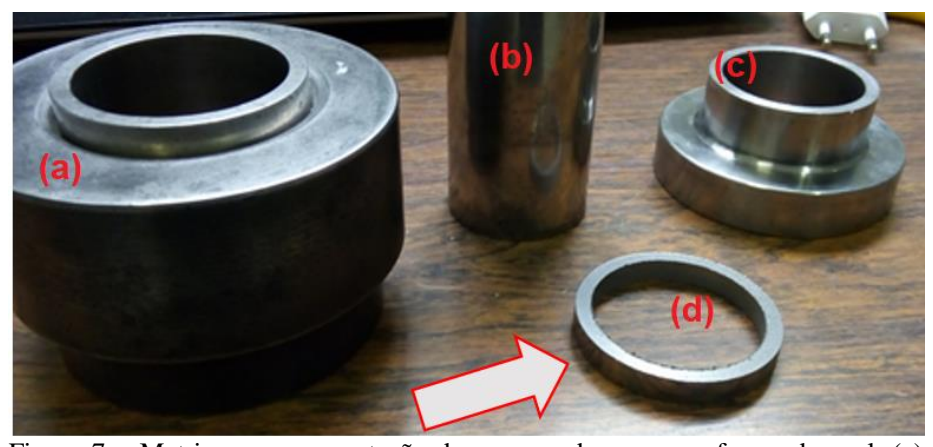

Figura 7 - Matriz para compactação dos corpos de prova na forma de anel. (a) matriz externa; (b) matriz interna; (c) punção superior; (d) anel compactado.

\subsection{Medição dos parâmetros eletromagnéticos}

Depois da preparação (bobinamento) da amostra, o ensaio para a obtenção da curva de histerese e magnetização é feito ainda com base na norma ASTM A773/A 773M-01 [16], sendo utilizado um equipamento apropriado (FIGURA 8), e um software para a aquisição dos dados (FIGURA 9), modelo TLMP-FLX-D, da empresa Global Mag.

Durante os ensaios de curva de magnetização do material, também conhecido como ensaio CC, foram utilizadas correntes de excitação de $5 \mathrm{~A}$ com frequência de $0,05 \mathrm{~Hz}$. Já para a obtenção da curva e histerese foram utilizadas frequência na faixa de $60 \mathrm{~Hz}$.

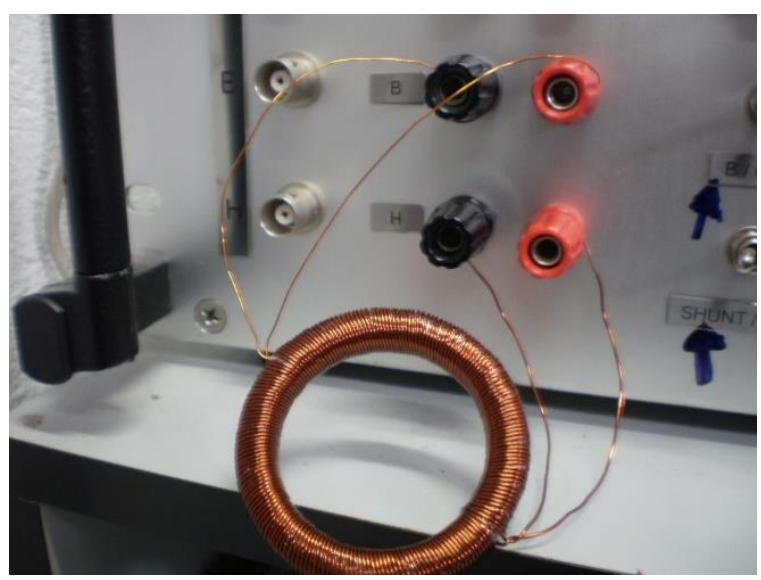

Figura 8 - Método de medição da curva de histerese pelo método do anel.

As curvas de histerese obtidas foram típicas de materiais magnéticos macios, sendo que destas curvas é possível retirar-se propriedades magnéticas importantes como a indução de saturação, magnetismo remanente e força coercitiva. A indução de saturação ou indução máxima, pode ser visualizada a partir dos valores de campo onde a indução tende a permanecer constante ou com declividade quase nula. 


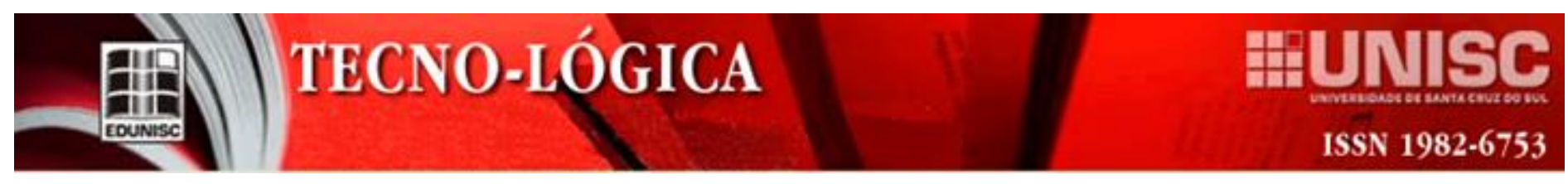

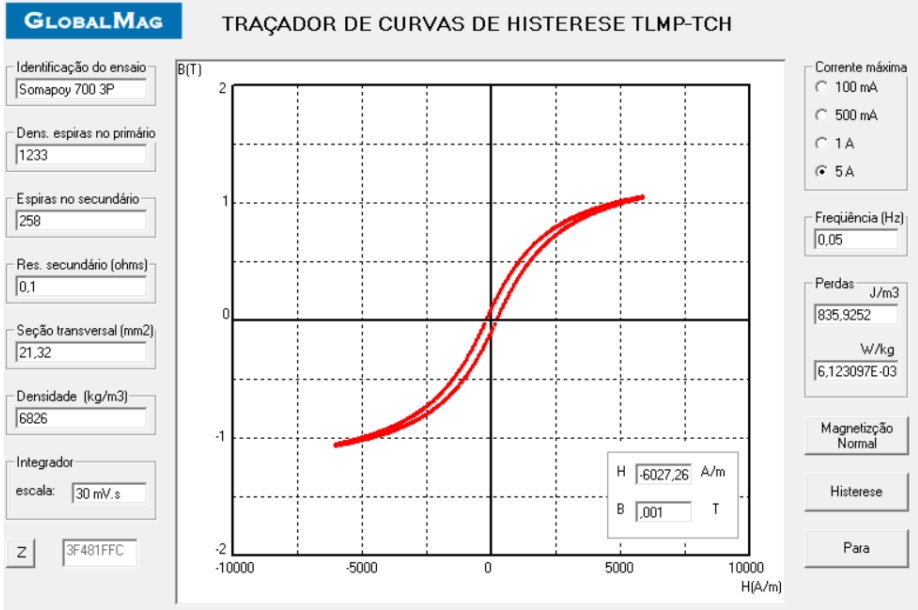

Figura 9 - Medição da curva de histerese pelo método do anel.

\subsection{Simulação via FEMM}

Como proposto neste trabalho, foi criado um ambiente de simulação para testar o material sinterizado a partir dos dados adquiridos nas curvas de histerese e magnetização. Para tanto, foi necessário do desenho em CAD do motor que se quer simular, criar um novo material no FEMM 4.2 [17], e inserir os parâmetros coletados nos testes magnéticos. A Figura 10 mostra esse procedimento, no qual os dados $B$ (fluxo magnético) e $H$ (intensidade de campo), que correspondem à curva de magnetização do material, são inseridos e salvos no programa. Além destes dados, informações como resistividade elétrica do material, condutividade entre outros dados também podem ser inseridos caso seja necessário.

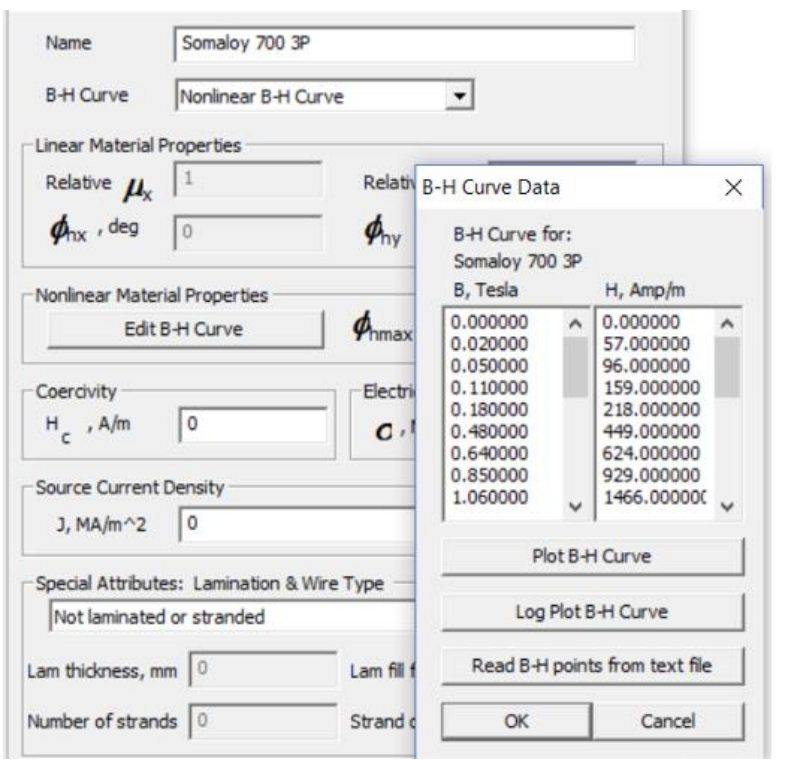

Figura 10 - Inserção dos parâmetros magnéticos do material Somaloy $7003 \mathrm{P}$.

\section{Resultados e discussões}

A simulação de materiais magnéticos auxilia no levantamento dos parâmetros eletromagnéticos do material tendo em vista a trabalhosa tarefa de concepção dos novos corpos de prova. Desta forma, o estudo do comportamento magnético de um SMC a partir de uma imagem metalográfica proporciona uma rápida análise e possibilita mudanças de parâmetros de forma a encontrar uma composição de liga e características mecânicas como maior ou menor compactação, determinação de tempos de tratamento térmico e possíveis modificações no design da peça.

\subsection{Simulação eletromagnética de materiais SMC}

Um dos objetivos deste trabalho foi o de determinar o comportamento da densidade de fluxo magnético $(B)$ para materiais compósitos sinterizados. Para tanto, utilizou-se como referência uma imagem da estrutura cristalográfica obtida de uma das amostras produzidas neste estudo (FIGURA 11-a), composta pelo SMC Somaloy 700 3P.

Inicialmente um tratamento digital da imagem foi necessário (FIGURA 11-b) para então ter um novo arquivo binarizado contendo os contornos de cada um dos grãos (FIGURA 11-c). Em seguida, foi possível dar início ao processo de criação do modelo CAD da imagem (FIGURA 11-d) e posterior simulação deste material.
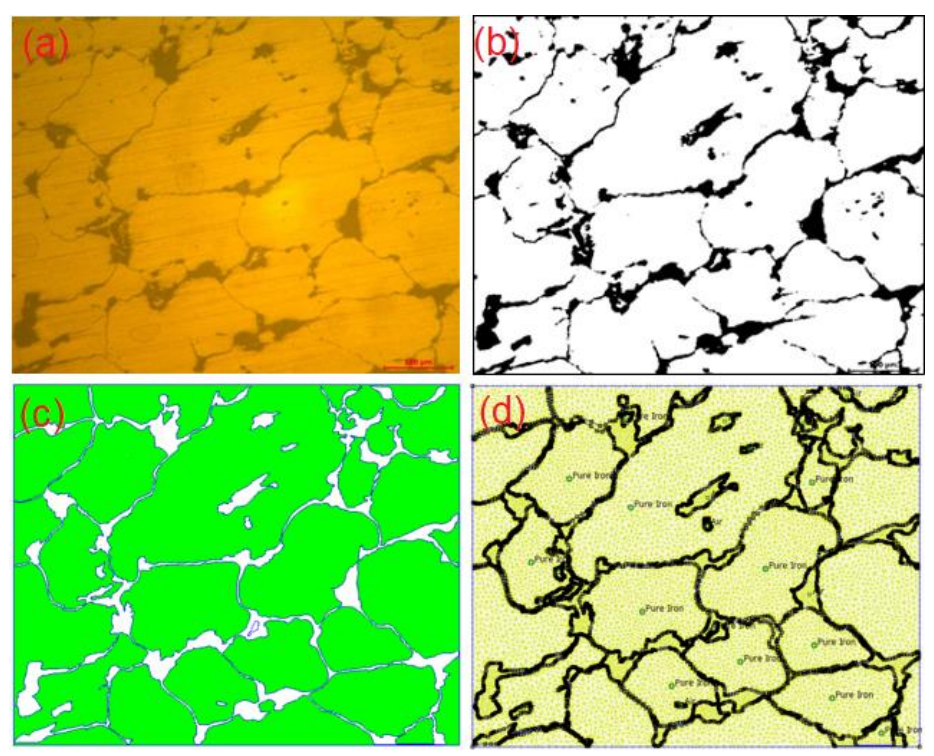

Figura 11 - (a) Imagem metalográfica do Somaloy $7003 \mathrm{P} \mathrm{e} \mathrm{(b)} \mathrm{imagem} \mathrm{binarizada.}$ (c) Grãos em verde e poros/isolamento em branco. (d) Os grãos são nomeados como ferro puro e os poros/isolamento como ar. 


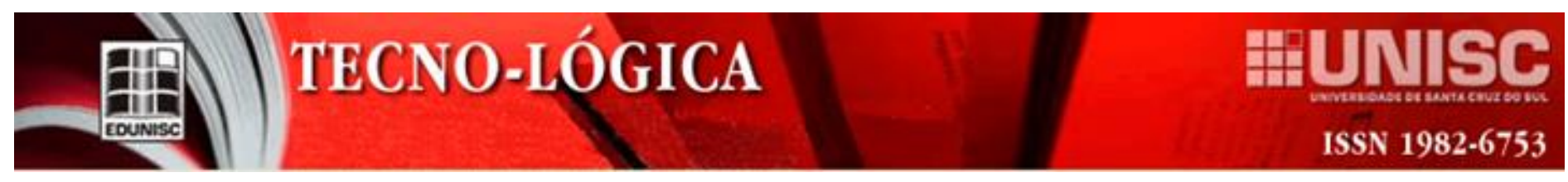

Os grãos podem ser visualizados na Figura 11-c, em verde, sendo que seus tamanhos variam de 70 um até 125 um aproximadamente. Já a Figura 11-d mostra o processo de nomeação de cada área (área composta por toda a extensão do contorno), e a criação da rede (mesh) utilizada pelo método de elementos finitos do software FEMM [17].

A etapa de nomeação das regiões que serão simuladas no FEMM consiste em definir os materiais que consiste na amostra. Assim sendo, os grãos são definidos como ferro puro (pure iron) e os poros, ou vazios, como regiões com presença de ar. Para a geração de um campo magnético que possa fluir pelo material, dois imãs permanentes são introduzidos na simulação, sendo o polo sul na parte superior e o polo norte na parte inferior da figura.

Também foram simuladas outras situações modificando o material da amostra por um bloco contendo apenas os dados da curva $B . H$ apresentada na Figura 10 . Esta simulação possibilita uma comparação entre os dois métodos, e também possibilita a construção de modelos computacionais reais de motores, geradores e transformadores.

Em relação aos resultados obtidos após a compilação do primeiro modelo, pode-se observar inicialmente como ocorre o fluxo magnético entre cada partícula do material ferromagnético (FIGURA 12). Além disso, diferentes parâmetros foram analisados, como densidade de fluxo magnético $(B)$, intensidade de campo $(H)$, permeabilidade e a resistividade do material. A análise do fluxo magnético induzido em uma imagem metalográfica da amostra Somaloy $7003 \mathrm{P}$ pode ser observada na que mostra a circulação de um fluxo magnético $(B)$ de cima para baixo.

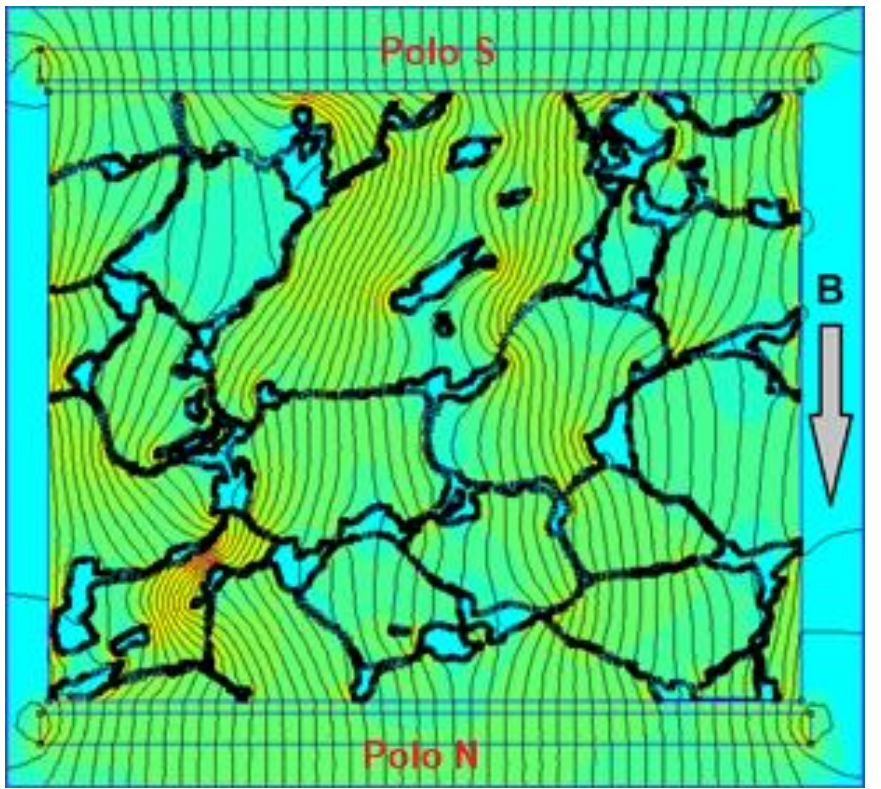

Figura 12 - Campo magnético aplicado de cima para baixo, criando um fluxo magnético induzido na vertical.
Já na Figura 13 foi feita a medição da densidade de fluxo magnético fluindo em cada uma das partículas de ferro. Foram 3 medições, onde a linha número 1 apresenta um $B$ igual a $0,19 \mathrm{~T}$. Na linha número 2 o $B$ corresponde a $0,76 \mathrm{~T}$ e a linha número 3 apresenta o maior valor de $B$, próximo a 1,7 Tesla, devido a uma considerável diminuição da largura do grão. Em algumas regiões há uma passagem de fluxo magnético reduzido devido ao fato de que a camada isolante do grão ser mais espessa, fazendo com que as linhas de fluxo optem por outros caminhos de menor resistência. Outro fator importante a ser observado refere-se à existência de poros (vazios), devido principalmente à pressão utilizada na prensagem da amostra.

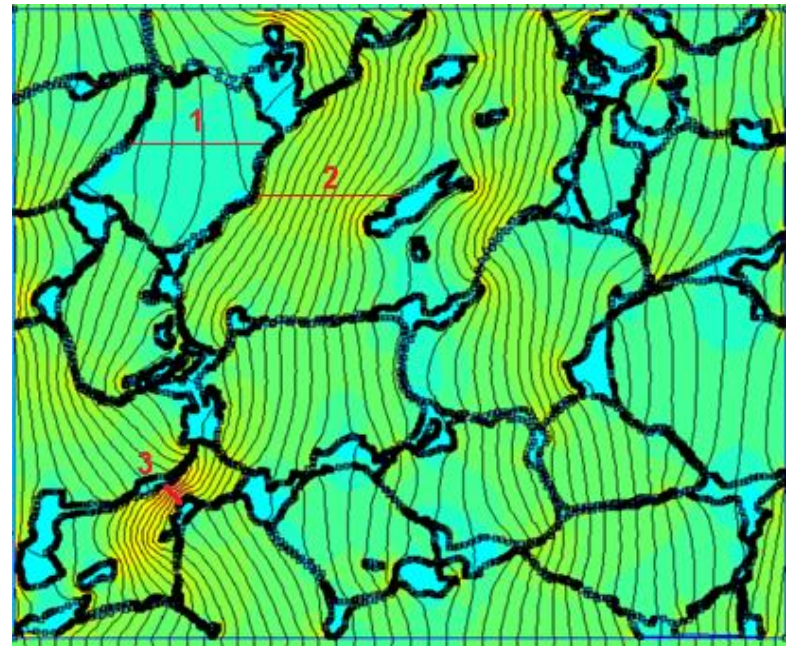

Figura 13 - Passagem de fluxo magnético em diferentes caminhos (grãos).

\subsection{Simulação eletromagnética de motores de indução}

A simulação de motores de indução tem o intuito de verificar o funcionamento do motor sem que este seja montado na prática. Os resultados levantados na simulação, comparados com os parâmetros já conhecidos, propiciam uma rota de estudo de forma a possibilitar ajustes nos materiais SMC sem que haja a necessidade da construção de um novo motor.

Assim, a Figura 14 mostra a simulação deste motor de indução trifásico, que é geralmente é montado com chapas laminadas típicas (M-19 Steel). No caso da substituição deste material pelo compósito magnético, de acordo com seus parâmetros de curva $B . H$, foi observar nos resultados de simulação que a densidade magnética máxima ficou próxima a 1,22 $\mathrm{T}$ para uma corrente de pico de 1 Amperes, ficando cerca de $17 \%$ menor que o modelo convencional (chapas laminadas). 


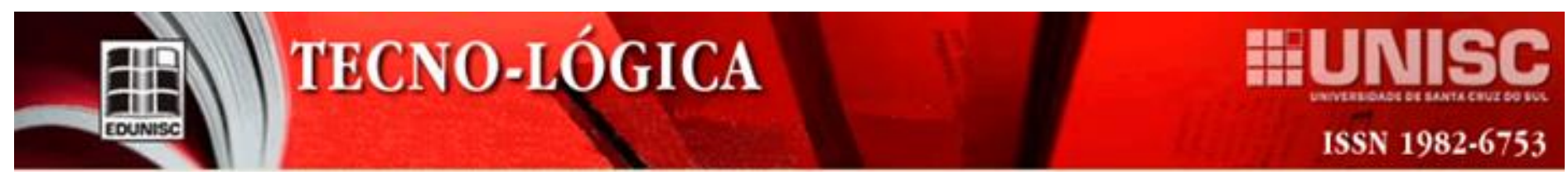

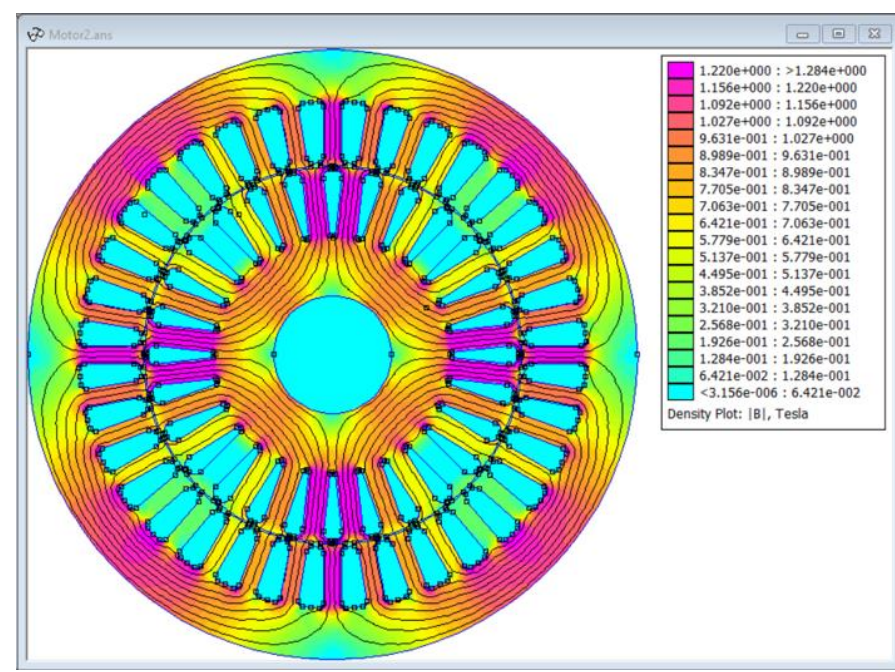

Figura 14 - Simulação de um motor utilizando os parâmetros do Somaloy 700 3P.

Além da densidade de fluxo magnético menor, SMC também apresentou uma permeabilidade magnética menor (ou seja, capacidade de condução de fluxo versus o campo magnético aplicado) e um aumento considerável da resistividade elétrica, chegando a $14 \mu \Omega$.m.

A partir destes dados, uma relação entre o material compósito e os convencionais pode ser realizada e as diferenças se justificam pela: (i) presença de poros (vazios) na amostra devido à falta de pressão na hora da compactação a amostra; (ii) a camada isolante muito espessa, em função da qualidade do recobrimento da partícula; e (iii) o aumenta a resistividade elétrica do material devido à grande quantidade de partículas que conduzem um valor de $B$ abaixo do valor de saturação $(1,22 \mathrm{~T})$ como apresentam as linhas 1 e 2 da Figura 13.

\section{Conclusões}

Neste estudo foram analisadas as propriedades eletromagnéticas de materiais SMC (Soft Magnetic Composites), criando assim um ambiente de simulação para testes em motores de indução trifásico. Por meio do desenvolvimento deste trabalho pode-se analisar as características dos compósitos magnéticos macios, tanto experimentalmente quanto por simulação, da liga Somaloy 700 3P, da empresa Höganäs AB.

A análise em fase amostral dos materiais foi utilizada para o levantamento das propriedades físicas e magnéticas, onde foi possível a comparação com valores obtidos na literatura, seja com trabalhos anteriores como também com a fabricante do material. Posteriormente, estes parâmetros foram inseridos em ambiente de simulação e o comportamento eletromagnético do material foi observado, além de um comparativo entre esta liga e os motores convencionais montados a partir de chapas laminadas.

Os resultados encontrados nas simulações apresentam um rendimento menor da liga Somaloy 7003 P, porém sua utilização um design em 3D que aumente a intensidade de campo magnético acarretará em um aumento considerável no fluxo magnético (B), superando assim os aços laminados. Outro fator importante que este estudo apresentou foi a análise do fluxo magnético baseado nas imagens metalográficas das amostras utilizadas nos testes. Estas imagens formas essenciais no entendimento do comportamento magnético dos SMCs, já que nas simulações pode-se observar que variáveis como tamanho de partícula, espessura da camada isolante e presença de poros podem afetar consideravelmente na relação $B . H$ da peça ou motor desenvolvido.

\section{USE OF COMPUTER SIMULATION FOR THE STUDY \\ OF ELECTROMAGNETIC PROPERTIES OF A SOFT MAGNETIC COMPOSITE (SMC) FOR FUTURE APPLICATION IN INDUCTION ENGINES}

ABSTRACT: This study presents the development of a method of computer simulation for the study of electromagnetic properties of a composite material for application in induction engines (rotor and stator), driven by the possibility of application of the SMC (Soft Magnetic Composites) materials as an alternative to the traditional electric steels (non-oriented grain laminate). In this way, the aim of the study was to develop a computer simulation environment to facilitate the survey of electric and magnetic parameters of an induction engine without the necessity of building a physic model. For this task, it was used the software FEMM 4.2 for the model simulations in $2 \mathrm{D}$, as well as to validate the parameters collected in comparison to commercial material of the same composition. A correlation between the magnetic flux circulation of the SMC materials and the laminated sheets was made, in which was observed that the composite material sample had properties compatible to laminated steels, however the electric resistivity was higher because of the thicker insulating particle layer. As final result, it is possible to set up a simulation method to facilitate the research and development of different Fe powder alloys, that can be used in new topologies of electric induction engines for miscellaneous industrial applications.

Keywords: Soft magnetic material. Induction engines. Magnetic flux.

\section{Referências}

[1] ISHIZAKI, T. et al. Improving Powder Magnetic Core Properties via Application of Thin, Insulating Silica-Nanosheet Layers on Iron Powder Particles. Nanomaterials, Vol. 7, n. 1, p. 1, 2016. 
[2] SHOKROLLAHI, H.; JANGHORBAN, K. Soft Magnetic Composite Materials (SMCs). Journal of Materials Processing Technology. 2007, Vol.189, pp. 1-12.

[3] DIAS, M. M., et al. Aplicação de materiais magnéticos macios sintetizados em máquinas elétricas rotativas síncronas. 2014. Tecnologia e Tendências. FEEVALE. Vol.10, $\mathrm{N}^{\circ} 2,2^{\circ} 2014$.

[4] UENO, T. et al. Practical and Potential Applications of Soft Magnetic Powder Cores with Superior Magnetic Properties. SEI TECHNICAL, n. 82, 9, 2016.

[5] CAMPOS, L. et al. Caracterização das propriedades magnéticas de materiais compósitos magnéticos macios. Revista de Iniciação Científica. ULBRA, RS, 2014

[6] LANDGRAF, F. J. G. et al. Propriedades Magnéticas de aços para fins elétricos. Aços: perspectivas para os próximos, Vol. 10, p. 109-128, 2002.

[7] DIAS, M. M.; et al. Novas Perspectivas das Máquinas Elétricas Trifásicas a Partir da Utilização de Materiais Magnéticos Macios Sinterizados. Novo Hamburgo: Revista Liberato, Vol. 6, n. 6, 2005. p. 56-65.

[8] GUIMARÃES, F. R. Desenvolvimento de uma Máquina Síncrona Trifásica com Ímãs de Nd-Fe-B e Núcleo do Estator a partir de Fe2\%P Sinterizado Aplicado em aerogerador de 1kW. 2015. Dissertação de Mestrado - UFRGS, 2015.

[9] NIADA, R. F. Desenvolvimento de um mini-motor com estator produzido por processo de metalurgia do pó. 2015. Tese de Doutorado - UFRGS, 2015.

[10] RONCHI, F. P. Construção e análise do desempenho de um motor de corrente continua utilizando materiais magnéticos macios a partir da metalurgia do pó. 2015. Dissertação de Mestrado - UFRGS, Porto Alegre, 2015.

[11] TONTINI, G. Estudo de compósitos magnéticos moles de ferro recoberto por suspensão de nanopartículas de alumina em vidro líquido. Tese de doutorado Universidade Federal de Santa Catarina, Programa de Pós-Graduação em Ciência e Engenharia de Materiais, Florianópolis, 2017.

[12] GRIFFITHS, D. J.; COLLEGE, R. Introduction to Electrodynamics. USA; New Jersey, Vol. 4, 2012.

[13] CULLITY, B. D.; GRAHAM, C. D. Introduction to Magnetic Materials. 2nd. New Jersey: John Wiley \& Sons, Inc., 2009. ISBN 978-0-471-47741-9.

[14] KOLLAR, P., et al. Power loss separation in Fe-based composite materials. Journal of Magnetism and Magnetic Materials. 2013, 327, pp. 146-150.

[15] HOGANAS, A. B. Compostos Magnéticos Macios (Soft Magnetic Composites - SMC). Disponível em: <https://www.hoganas.com/>.

[16] NORMA ASTM A773/A 773M-01 - Standard Test Method for Direct Current Magnetic Properties of Low Coercivity Magnetic Materials Using Hysteresigraphs

[17] FEMM. Finite Element Method Magnetics. Disponível em: <http://www.femm.info/wiki/HomePage> 